\title{
Molecular Recognition and Conductance in Crown Ethers
}

\author{
Chris Liu, Derek Walter, and Daniel Neuhauser \\ Department of Chemistry and Biochemistry, University of California, Los Angeles, California 90095 \\ Roi Baer \\ Institute of Chemistry, and the Lise Meitner center the Hebrew University of Jerusalem, Jerusalem, 91904 Israel \\ Received October 24, 2002; E-mail: Roi_Baer@huji.ac.il
}

Crown ethers have the remarkable property of recognizing and binding specific metal cations in complex mixtures. Consequently, since their discovery, ${ }^{1}$ they have found numerous applications in science and industry and stimulated the field of molecular recognition and host-guest chemistry. One area remaining unexplored is the conductance properties of crown ethers. Of particular interest is the change in conductance as the crown binds and relinquishes a cation. Such a chemical system could possibly be used as a molecular sensor or a switch. These questions are, in fact, accessible to experimentalists. Recent synthetic advances enable researchers to form molecular wires (MWs) by binding elongated organic molecules to metallic leads such as gold. The conductance of these MWs is readily measured as their current-voltage characteristics. ${ }^{2-7}$ This has led to the development of various new prototypes of molecular scale electronic devices, ${ }^{8}$ among them, rectifiers, ${ }^{9,10}$ memory devices, ${ }^{11}$ switches, ${ }^{12}$ and transistors. ${ }^{6}$

This communication combines molecular recognition and molecular electric conductance to form a single electronic device. On a slightly larger scale, such an attempt was recently successful, ${ }^{13}$ where biotin molecules functionalized a silicon nanowire, and conductance dropped by $12 \%$ upon exposure to m-antibiotin.

Here, we study a short molecular wire containing a crown-6 molecule connected via sulfur atoms to two long atomic gold wires (AGW), as shown in Figure 1. This is a realistic system since AGWs are perfect quantum conductors. ${ }^{14}$ It is possible that the present model has a wider scope of applicability, beyond AGWs. The wires have an average HOMO-LUMO energy of $-6.49 \mathrm{eV}$, in close proximity to ab initio computations of the bulk gold ${ }^{15}$ and gold clusters $^{16}$ work functions. Furthermore, there are indications that conductance in organic MWs connected by sulfur to gold surfaces is insensitive to the geometry of the latter. ${ }^{17}$

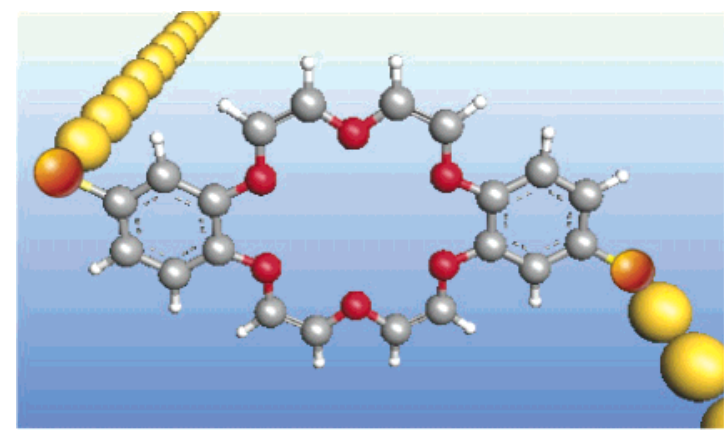

Figure 1. MW-containing crown-6 connected via sulfur atoms (orange) to atomic wires of gold (yellow). The cation binds electrostatically into the negatively charged region surrounded by the 6 oxygen (red) atoms of the crown.

The molecular geometries, with and without the metallic cations, were determined by molecular mechanics methods, ${ }^{18}$ known to yield

13936 | J. AM. CHEM. SOC. 2003, 125, 13936-13937 reasonable results. ${ }^{19}$ We then launched a density functional theory (DFT) calculation, with the B3LYP functional and 6-31G*/ LACVP* effective core potentials (using Jaguar program). To test the adequacy of this DFT, we calculated the gas-phase binding energies and found good agreement with recent measurements (Table 1).

Table 1: Binding Energies (eV) of Cations to C-6 (Figure 1 without $S$ and $\mathrm{Au}$ Atoms $^{a}$ )

\begin{tabular}{lcccl}
\hline cation & $\begin{array}{c}\text { B3LYP } \\
\text { LACVP }\end{array}$ & $\begin{array}{c}\text { B3LYP } \\
\text { LACVP* }\end{array}$ & MP220 & $\begin{array}{c}\text { gas-phase } \\
\text { experiment }\end{array}$ \\
\hline $\mathrm{H}+$ & 6.8 & 6.8 & $\mathrm{n} / \mathrm{a}$ & $\mathrm{n} / \mathrm{a}$ \\
$\mathrm{Li}+$ & 4.2 & 3.7 & 4.2 & $\mathrm{n} / \mathrm{a}$ \\
$\mathrm{Na}+$ & 3.0 & 2.7 & 3.5 & $3.05(0.19)$ \\
$\mathrm{K}+$ & 2.1 & 1.8 & 3.1 & $2.12(0.15)$ \\
\hline
\end{tabular}

${ }^{a}$ B3LYP are present results using two basis sets.

With the DFT computation, one obtains an excellent zero-order Hamiltonian that describes the unbiased MW. The zero-bias conductance should now be calculated using time-dependent DFT. 22 Yet, at present, this method is not well developed and is too timeconsuming for the present system. An alternative approach is to use the noninteracting particle model $^{23}$ of Landauer, by which the current $I$ associated with a voltage bias $V$ is:

$$
\begin{aligned}
& I=2 e^{2} / h \int\left\{\left(1+\exp \left[\beta\left(E-\mu^{L}\right)\right]\right)^{-1}-\right. \\
& \left.\left(1+\exp \left[\beta\left(E-\mu^{R}\right)\right]\right)^{-1}\right\} T(E) \mathrm{d} E
\end{aligned}
$$

Here, $\beta$ is the inverse temperature (room temperature in present calculations) and $\mu_{i}\left(\mu_{i}=L, R\right)$ are the chemical potentials (including voltage bias) of the two leads. The cumulative transmission probability $T(E)$ ("transmittance") is calculated using methods, introduced by others in this field, ${ }^{24-29}$ which we briefly describe here. To the Kohn-Sham Fock-matrix $F_{\mathrm{KS}}$ (calculated for the optimized geometries), we add two absorbing potentials stretched along the two gold wires. This results in a complex Fock matrix $F$ $=F_{\mathrm{KS}}-i\left(\Gamma^{L}+\Gamma^{R}\right)$. Here, $\Gamma^{L}$ and $\Gamma^{R}$ are positive diagonal matrices in the atomic orbital basis located on the left and right gold leads. These potentials allow the computation of quantum transmittance using techniques adopted from reaction scattering theory. ${ }^{30}$ The transmittance at energy $E$ can be determined by the SeidemanMiller formula: ${ }^{31}$

$$
T(E)=4 \operatorname{Tr}\left\{G^{\dagger}(E) \Gamma^{L} G(E) \Gamma^{R}\right\}
$$

where $G(E)=(E-F)^{-1}$. This model is appropriate only for small bias since the Hamiltonian is independent of the bias. Some information on the reliability of the method is available. For some systems it has been tested against experiments, and the present experience is that the absolute conductance is grossly overestimated 
by the method, yet the shape of the $I(V)$ curves is often reproduced. ${ }^{27,28}$ Not being able to use the more rigorous TDDFT method, we resort to the Landauer-DFT approach in the present work.

The conductance of the molecular wires in three binding situations is shown in Figure 2. Molecular wires containing a

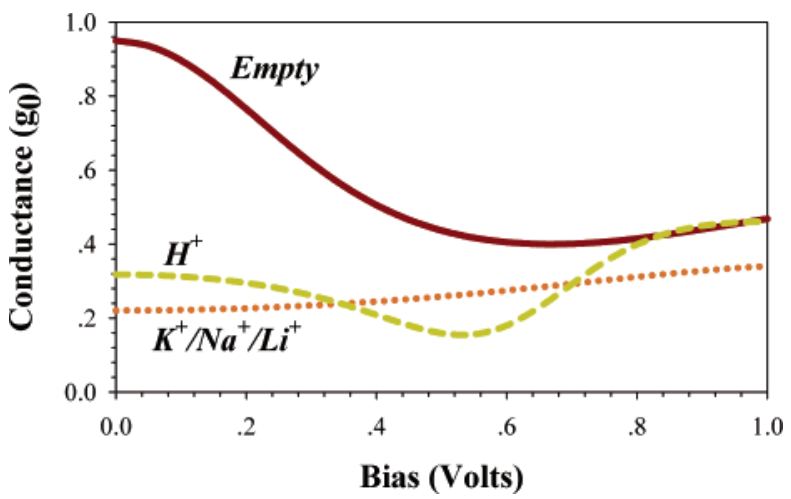

Figure 2. Low bias conductance of crown-6 systems. $g_{0}=2 e^{2} / h$.

potassium cation show weakest conductance. The curve for sodium and lithium cations (not shown) is identical to that of potassium, but proton behaves differently. It is clear that the system with an empty crown conducts significantly better than a loaded one.

To better understand the conduction pattern we examine the transmittance and density of states (DOS) curves $\rho(E)=\pi^{-1}$ $\operatorname{Im} \operatorname{Tr} G(E)$, in the vicinity of the Fermi level in Figure 3. All three systems exhibit a similar DOS at the Fermi level of about 10 states per $\mathrm{eV}$ (i.e. a HOMO-LUMO spacing of $\sim 0.1 \mathrm{eV}$ ). This is due to the fact that the crown-ligand interaction is mostly electrostatic, involving little charge transfer. ${ }^{20}$ The high DOS is a necessary condition for high conductance. Indeed, the empty crown conducts very well at zero bias, with transmission probability $T\left(\epsilon_{F}\right) \approx 1$. But clearly, high DOS is not a sufficient condition. For conductance, one also needs extended states. The proton-loaded crown exhibits an anti resonance (AR) in the transmission coefficient almost exactly at the Fermi energy. This means that there are no extended states at this energy, causing the low conductance seen for zero bias (Figure 2) followed by a gentle dip of conductance at $0.5 \mathrm{eV}$. As the bias is further raised, the effect of the AR is diminished, and conductance grows rapidly. This is because $T(E)$ rises steeply as energy is lowered bellow the location of the AR. ARs in MWs were first discussed in detail by Emberly et al. ${ }^{32}$ ARs are due to destructive interference having strong effects on the conductance of some organic MWs. ${ }^{33-36}$ When a larger cation is bound to the crown, an AR is not visible, but the interference effect is present and smeared over a larger energy interval (Figure 3). This leads to reduced transmittance monotonically increasing with bias in Figure 2.

Summarizing, the cationic binding significantly lowers conductance due to quantum mechanical interference effects. A striking insensitivity of conductance to the type of ligand is predicted. Experimental work is now required to determine the extent to which these calculated effects can be felt in practice.

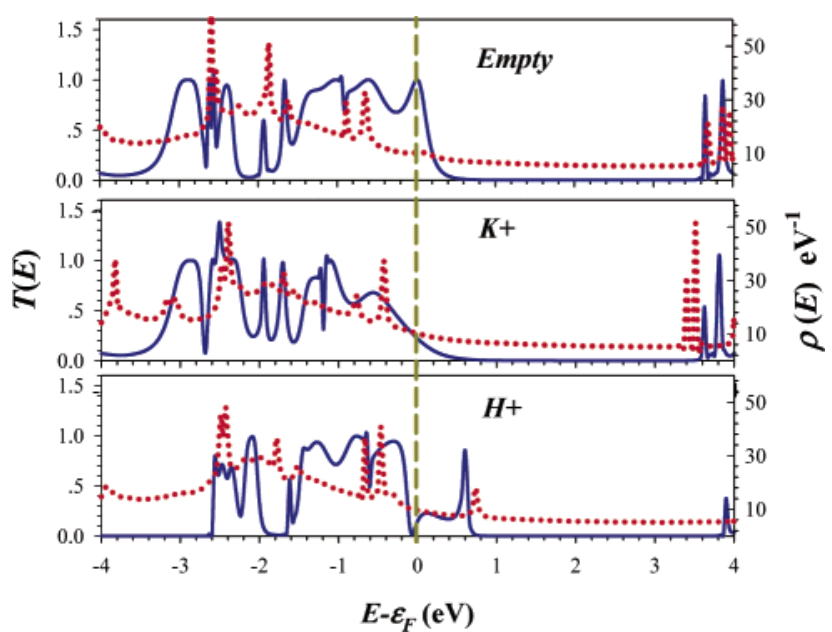

Figure 3. Transmittance $T(E)$ (solid) and DOS $\rho(E)$ (dotted) vs energy (from $E_{\mathrm{F}}=\left(E_{\mathrm{HOMO}}+E_{\mathrm{LUMU}}\right) / 2$ ) for empty or $\mathrm{K}^{+} / \mathrm{H}^{+}$-loaded crown.

Acknowledgment. We thank E. Rabani, M. Ratner, and J. Zink for discussions. Work is supported by Israel Science Foundation, NSF, and PRF. D.W. acknowledges UCLA Dissertation Year Fellowship.

\section{References}

(1) Pederson, C. J. J. Am. Chem. Soc. 1967, 89, 2495.

(2) Reed, M. A., et al. Science 1997, 278, 252.

(3) Kergueris, C., et al. Phys. Rev. B 1999, 59, 12505.

(4) Chen, J., et al. Science 1999, 286, 1550.

(5) Tour, J. M., et al. Chem.-Eur. J. 2001, 7, 5118.

(6) Park, J., et al. Nature 2002, 417, 722.

(7) Zhitenev, N. B., et al. Phys. Rev. Lett. 2002, 88, 226801

(8) Aviram, A.; Ratner, M. A. Chem. Phys. Lett. 1974, 29, 277.

(9) Metzger, R. M. Synth. Met. 2001, 124, 107.

(10) Fischer, C. M., et al. Synth. Met. 1995, 71, 1975

(11) Luo, Y., et al. ChemPhysChem 2002, 3, 519.

(12) Pease, A. R. Jeppesen, J. O.; Stoddart, J. F.; Luo, Y.; Collier, C. P.; Heath, J. R. Acc. Chem. Res. 2001, 34, 433

(13) Klemic, J. F., et al. Nat. Biotechnol. 2001, 19, 924.

(14) Ohnishi, H., et al. Nature 1998, 395, 780.

(15) Skriver, H. L.; Rosengaard, N. M. Phys. Rev. B 1992, 46, 7157.

(16) Weber, H. B., et al. Chem. Phys. 2002, 281, 113.

(17) Yaliraki, S. N.; Kemp, M.; Ratner, M. A. J. Am. Chem. Soc. 1999, 121 3428.

(18) Rappe, A. K.; Casewit, C. J.; Colwell, K. S.; Goddard, W. A., III; Skiff, W. M. J. Am. Chem. Soc. 1992, 114, 10024.

(19) Thompson, M. A., et al. J. Phys. Chem-Us 1994, 98, 10465.

(20) Glendening, E. D.; Feller, D.; Thompson, M. A. J. Am. Chem. Soc. 1994 $116,10657$.

(21) More, M. B.; Ray, D.; Armentrout, P. B. J. Am. Chem. Soc. 1999, 121 417.

(22) Baer, R.; Neuhauser, D. Int. J. Quantum Chem. 2003, 91, 524.

(23) Baer, R.; Neuhauser, D. Chem. Phys. Lett. 2003. In press.

(24) Nitzan, A. Annu. Rev. Phys. Chem. 2001, 52, 681.

(25) Yaliraki, S. N., et al. J. Chem. Phys. 1999, 111, 6997.

(26) Damle, P., et al. Chem. Phys. 2002, 281, 171.

(27) Stokbro, K., et al. Comput. Mater. Sci. 2003, 27, 151.

(28) Di Ventra, M., et al. Phys. Rev. Lett. 2000, 84, 050979.

(29) Xue, Y. Q., et al. Chem. Phys. 2002, 281, 151.

(30) Neuhasuer, D.; Baer, M. J. Chem. Phys. 1989, 90, 4351

(31) Seideman, T.; Miller, W. H. J. Chem. Phys. 1992, 96, 4412.

(32) Emberly, E. G.; Kirczenow, G. J. Phys. C 1999, 11, 6911.

(33) Sautet, P.; Joachim, C. Chem. Phys. Lett. 1988, 153, 511.

(34) Baer, R.; Neuhasuer, D. Chem. Phys. 2002, 281, 353.

(35) Baer, R.; Neuhauser, D. J. Am. Chem. Soc. 2002, 124, 4200.

(36) Walter, D., et al., submitted 2002. JA029085P 